\title{
IMPLEMENTATION OF THE INTEGRATED CARE OF OLDER PEOPLE (ICOPE) APP IN PRIMARY CARE: NEW TECHNOLOGIES IN GERIATRIC CARE DURING QUARANTINE OF COVID-19 AND BEYOND
}

\author{
D. SANCHEZ-RODRIGUEZ ${ }^{1,2}$, C. ANNWEILER ${ }^{3,4}$, S. GILLAIN ${ }^{5}$, B. VELLAS 6
}

\begin{abstract}
1. WHO Collaborating Centre for Public Health Aspects of Musculoskeletal Health and Aging, Division of Public Health, Epidemiology and Health Economics, University of Liège, Liège, Belgium; 2. Geriatrics Department, Rehabilitation Research Group, Hospital Del Mar Medical Research Institute (IMIM), Universitat Pompeu Fabra, Barcelona, Spain;

3. Department of Neurosciences and Aging, Division of Geriatric Medicine, Angers University Hospital; Angers University Memory Clinic; Research Center on Autonomy and Longevity; UPRES EA 4638, University of Angers, UNAM, Angers, France; 4. Robarts Research Institute, Schulich School of Medicine and Dentistry, University of Western Ontario, London, Ontario, Canada; 5. Geriatrics Department, Liège University Hospital, Liège, Belgium; 6. Department of Geriatrics, Toulouse University Hospital; Gerontopole of Toulouse, INSERM U1027, University of Toulouse III, Toulouse, France.

Corresponding author: Dolores Sanchez-Rodriguez, MD PhD. WHO Collaborating Centre for Public Health Aspects of Musculoskeletal Health and Aging, Division of Public Health, Epidemiology and Health Economics, University of Liège, CHU - Sart Tilman, Quartier Hôpital, Avenue Hippocrate 13 (Bât. B23), Liège, Belgium, Tel: +32 43 66 2812 and +32 4934327 50, Emails: dolores.sanchez@uliege.be and dolo.sanchezr@gmail.com
\end{abstract}

J Frailty Aging 2021;10(2)139-140

Published online May 6, 2020, http:/ / dx.doi.org/10.14283/jfa.2020.24

"I could create all sorts of machines, both for the protection of the city and for siege in times of war and for creating water channels and modifying the course of rivers in times of peace"

Letter from Leonardo Da Vinci to the Lord of Milan, 1482

The COVID-19 pandemic due to a novel coronavirus (SARS-CoV-2) in December 2019 has rapidly spread worldwide. The mortality rate is about $2.3 \%$ in general population, with high human-to-human transmission of 0.41 (credible interval $[0.27,0.55]$ ), and nasopharyngeal asymptomatic carriers act as vectors within the population (1). The World Health Organization (WHO) declared the pandemic on March 2020, and established objectives and action plan. First, WHO aimed at limiting the transmission of SARS$\mathrm{CoV}-2$, which required large isolation actions (country borders lockdown and individual quarantine). Second, WHO aimed at guiding and supporting the different health care systems across countries. Finally, developing therapeutic interventions appeared as a global priority as available evidence were still scarce (1). More than 860 clinical trials are ongoing worldwide.

Despite all age groups are similarly affected by COVID19 , older adults experience a higher risk of developing severe complications, with mortality raising up to $8 \%$ in patients 70 -79-year-old, and up to $14.8 \%$ in those aged $\geq 80$ years. Europe is particularly concerned by this problem due to the aging of the population. In 2016, the European Union counted 98 million people aged 65 years or more, compared to only 80 million children aged 16 years or less (https:/ec.europa. eu/eurostat/statistics-explained/index.php). Moreover, it is noticeable that the isolation measures have reduced the availability of the primary care givers and led to delayed/ cancelled outpatients' clinics, which increases the risk of acute decompensation of chronic diseases and frailty process in shortand mid-terms, independently of the COVID-19 infection. In this context, implementing new technologies based on quantified-self and on-line resources may help optimizing older adults' health.

Received April 22, 2020

Accepted for publication April 27, 2020
The Integrated Care of Older People (ICOPE) project is an international program $(2,3)$, based on the measurement of "Intrinsic capacity", a new concept defined by the World Health Organization plan of action 2016-2020, as "the composite of all the physical, functional, and mental capacities of an individual" (3-6) Intrinsic capacity changes the focus from a negative paradigm of aging (diseases, disability) towards a positive focus related to "optimal aging" $(4,6)$. Intrinsic capacity is focused on disease prevention, it has been related to the ability of individuals to overcome stressful or acute events $(4,6)$. Intrinsic capacity has been recently associated with the onset of autonomy decline, falls, and death during a 3-year follow-up (5). In a second step in the long-term, the ICOPE project is intended to collect large population data for the development of "Nomograms" of optimal aging in older adults.

Several actions to implementation of the ICOPE program in the community have been scheduled by WHO (2), and one is the ICOPE App, a free-of-charge App (Android/Apple Store https://www.youtube.com/watch?v=gLva4ReV9KA. The first version of the ICOPE App is available and allows a selfadministered screening of individuals' intrinsic capacity. New functions of the App are developed and include comprehensive geriatric assessment, sharing on-line information, and receive individual guide and support from primary care.

The implementation of the ICOPE App would mitigate the current gap between geriatricians and family physicians and those between the family physicians and the patients. Moreover, the decline in intrinsic capacity measured with the App may be useful to anticipate clinical adverse outcomes, such as prefrailty, missteps, falls, disability (i.e., a 20-point Barthel score), institutionalization, decreased quality of life, and death) and to share professional advices to community-dwelling older adults.

Some research teams are already following WHO guidelines in the implementation of the ICOPE App $(2,3)$, which needs:

1) Partnership with primary care physicians to share the App and ensure the feasibility of the App in each environment (e.g. by the Technology, Economics, Legal, Operational, Scheduling 


\section{IMPLEMENTATION OF THE INTEGRATED CARE OF OLDER PEOPLE (ICOPE) APP IN PRIMARY CARE}

-TELOS- model).

2) Evaluation of the association between the components of intrinsic capacity at baseline and the incidence of adverse health consequences in prospective cohort studies. It would be interesting to evaluate the relationship among intrinsic capacity at baseline and incidence of frailty, prefrailty, falls, disability, and decreased quality of life, hospitalization, institutionalization and particularly, if the score at baseline measured by the App is associated with all-cause mortality including mortality related to COVID-19.

3) Assessment of the impact of the ICOPE project and App on community-dwelling older adults and revisiting strategies towards geriatricians and primary care physicians.

The results expected from the implementation of the ICOPE App would be i) to support primary care, and indirectly create a long-term clinical and research partnership; ii) for the older patients, to better identify those at risk of developing frailty and adverse health consequences, and to be able to make reasoned decisions in clinical routine; and iii) for the health system, to invest in tools likely to prevent decline in intrinsic capacity and maintain function late in life.

The ICOPE project has a number of strengths. The first one is that the project is timely, as there is an increasing, crucial need of providing support to Primary Care physicians and community-dwelling older patients; moreover, on-line health resources had raised in interest in the latest years (7) and now even more due to the COVID-19 pandemic. The implementation of the ICOPE project involves economic advantages because no additional materials are required to deliver support, with the exception of the human cost of the healthcare providers engaged. In addition, the ICOPE App is free-of-charge, suitable for all brands (Android/Phone) and devices (telephones, computers, etc.). Of course, online communication and technical devices required by the ICOPE App could be challenging for older adults. However, this limitation could be counteracted by taking the window of opportunity of the quarantine, which makes older people and their families more likely to get engaged in online communication. The new-acquired technical skills by the users are effective in controlling the environment e.g. receiving advice and professional support, therefore, the behavior is reinforced (8). Finally, it is noticeable that using the ICOPE App may be slightly modified during the COVID19 pandemic compared to the original aim, which was to measure individuals' intrinsic capacity in a non-pandemic "normal" situation. This is also a great opportunity to unleash the potential of academic discoveries (9).

Part of the Inspire program (9-11), the apps ICOPE-
MONITOR is also free available both with Android and iPhone, and give the possibility to monitor intrinsic capacities overtime. Connected to a secure medical database, the ICOPE STEP 1 is performed every 4-6 months by professionals or seniors themselves. If a deterioration in one or more domains of intrinsic capacity is identified, an alert is generated by an algorithm which allows the health professionals to intervene quickly (11).

In conclusion, the ICOPE App applies to the "Actionresearch philosophy"(12) to bridge the gap between research and clinical practice, and to provide better care for older community-dwellers, especially during this exceptional and dramatic period of pandemic and containment, after which the world of tomorrow will no longer look like the one before.

Conflicts of interest: Authors declare no conflict of interest.

Authors' contribution: DSR and CA conceived the manuscript; DSR, CA, and SG wrote the manuscript; DSR and SG did literature review; BV corrected the manuscript. All co-authors read and approved the final version of the manuscript.

\section{References}

1. Zhou F, Yu T, Du R, Fan G, Liu Y, Liu Z, et al. Clinical course and risk factors for mortality of adult inpatients with COVID-19 in Wuhan, China: a retrospective cohort study. Lancet (London, England). 2020 Mar 28;395(10229):1054-62.

2. Briggs AM, De Carvalho IA. Actions required to implement integrated care for older people in the community using the World Health Organization's ICOPE approach: A global Delphi consensus study. PLoS One. 2018 Oct 1;13(10):e0205533.

3. Thiyagarajan JA, Araujo de Carvalho I, Peña-Rosas JP, Chadha S, Mariotti SP, Dua $\mathrm{T}$, et al. Redesigning care for older people to preserve physical and mental capacity: WHO guidelines on community-level interventions in integrated care. PLOS Med. 2019 Oct 18;16(10):e1002948.

4. Beard JR, Officer A, de Carvalho IA, Sadana R, Pot AM, Michel J-P, et al. The World report on ageing and health: a policy framework for healthy ageing. Lancet. 2016 May 21;387(10033):2145-54.

5. Charles A, Buckinx F, Locquet M, Reginster J-Y, Petermans J, Gruslin B, et al Prediction of adverse outcomes in nursing home residents according to intrinsic capacity proposed by the World Health Organization. Newman A, editor. J Gerontol A Biol Sci Med Sci. 2019 Sep 28;

6. Cesari M, Araujo de Carvalho I, Amuthavalli Thiyagarajan J, Cooper C, Martin FC Reginster J-YY, et al. Evidence for the Domains Supporting the Construct of Intrinsic Capacity. J Gerontol A Biol Sci Med Sci. 2018 Feb 2;73(12):1653-60.

7. Sanchez -Rodriguez $D$, Bruyère $O$. The International Registry of patients with sarcopenia : applying research in sarcopenia to clinical practice . Eur Geriatr Med. 2018 Dec 17;9(6):735-8.

8. Karsh N, Eitam B. I control therefore I do: Judgments of agency influence action selection. Cognition. 2015 May 1;138:122-31.

9. De Souto Barreto P, Guyonnet S, Ader G, Andrieu S, Casteilla L, Davezac N, et al. The INSPIRE research initiative: A program for geroscience and healthy aging research going from animal models to humans and the healthcare system. J Frailty Aging. 2020;DOI:10.14283/jfa.2020.18.

10. Takeda C, Guyonnet S, Sumi Y, Vellas B, Araujo De Carvalho I, Prev J, et al. Original Research Integrated Care for Older People and the Implementation in the INSPIRE Study. J Prev Alzheimer's Dis 2020. 2020 Mar 2;7(2):1-5.

11. Tavassoli N, Piau A, Berbon C, De Kerimel J, Lafont C, De Souto Barreto P, et al. Framework Implementation of the INSPIRE ICOPE-CARE program in collaboration with the World Health Organization (WHO). J Frailty Aging. 2020;In Press.

12. Beauchet O, Fantino B, Annweiler C. The 'Action-Research' philosophy: from bedside to bench, to bedside again. Int J Clin Pract. 2012 May;66(5):517-517. 